\title{
Omega-3 Fatty Acid Status Enhances the Prevention of Cognitive Decline by B Vitamins in Mild Cognitive Impairment
}

\author{
Abderrahim Oulhaj $^{\mathrm{a}, *}$, Fredrik Jernerén ${ }^{\mathrm{b}}$, Helga Refsum ${ }^{\mathrm{b}, \mathrm{c}}$, A. David Smith $^{\mathrm{b}}$ \\ and Celeste A. de Jager, ${ }^{\mathrm{d}, *}$ \\ ${ }^{a}$ Institute of Public Health, College of Medicine and Health Sciences, United Arab Emirates University, \\ United Arab Emirates \\ ${ }^{\mathrm{b}}$ OPTIMA, Department of Pharmacology, University of Oxford, Oxford, UK \\ ${ }^{\mathrm{c}}$ Department of Nutrition, Institute of Basic Medical Sciences, Faculty of Medicine, University of Oslo, \\ Oslo, Norway \\ ${ }^{\mathrm{d}}$ Division of Geriatric Medicine, Department of Medicine, Faculty of Health Sciences, \\ University of Cape Town, South Africa
}

\begin{abstract}
A randomized trial (VITACOG) in people with mild cognitive impairment (MCI) found that B vitamin treatment to lower homocysteine slowed the rate of cognitive and clinical decline. We have used data from this trial to see whether baseline omega-3 fatty acid status interacts with the effects of B vitamin treatment. 266 participants with MCI aged $\geq 70$ years were randomized to B vitamins (folic acid, vitamins B6 and B12) or placebo for 2 years. Baseline cognitive test performance, clinical dementia rating (CDR) scale, and plasma concentrations of total homocysteine, total docosahexaenoic and eicosapentaenoic acids (omega-3 fatty acids) were measured. Final scores for verbal delayed recall, global cognition, and CDR sum-of-boxes were better in the B vitamin-treated group according to increasing baseline concentrations of omega- 3 fatty acids, whereas scores in the placebo group were similar across these concentrations. Among those with good omega-3 status, $33 \%$ of those on B vitamin treatment had global CDR scores $>0$ compared with 59\% among those on placebo. For all three outcome measures, higher concentrations of docosahexaenoic acid alone significantly enhanced the cognitive effects of B vitamins, while eicosapentaenoic acid appeared less effective. When omega-3 fatty acid concentrations are low, B vitamin treatment has no effect on cognitive decline in MCI, but when omega-3 levels are in the upper normal range, B vitamins interact to slow cognitive decline. A clinical trial of B vitamins combined with omega- 3 fatty acids is needed to see whether it is possible to slow the conversion from MCI to $\mathrm{AD}$.
\end{abstract}

Keywords: Alzheimer's disease, B vitamins, clinical dementia rating scale, cognition, omega-3 fatty acids

\footnotetext{
${ }^{*}$ Correspondence to: Celeste de Jager, PhD, Division of Geriatric Medicine, Department of Medicine, IAA, L51, Old Main Building, Groote Schuur Hospital, Observatory, 7925, Western Cape, South Africa. Tel.: +27 21406 6538; Fax: +27 021406 6846; E-mail: Celeste.DeJager@uct.ac.za. and Abderrahim Oulhaj, PhD, Institute of Public Health, College of Medicine and Health Sciences, United Arab Emirates University, United Arab Emirates. Tel.: +9713713 7461; Fax: +971 3767 2022; E-mail: aoulhaj@uaeu.ac.ae.
}

\section{INTRODUCTION}

Trials to delay or prevent cognitive decline and Alzheimer's disease with nutrients are increasingly relevant due to the lack of new drugs to treat older persons with cognitive impairment. It is likely that the earlier treatment or preventative measures are introduced, the better the resulting efficacy of intervention for those 
with memory impairment, since the loss of neural networks is not as great at early stages of cognitive decline as it is when dementia has become established.

Nutrients involved in different metabolic pathways that have shown some positive effects on cognition include B vitamins and omega-3 fatty acids [1-3]. Inadequate $B$ vitamin status results in accumulation of homocysteine, a non-essential amino acid. Elevated plasma total homocysteine (tHcy) is a recognized modifiable risk factor for cognitive impairment, Alzheimer's disease [4], and other dementias [5]. Treatment trials with B vitamins have given conflicting results on cognitive outcomes [6-8], but some trials carried out specifically on people with elevated tHcy concentrations have shown positive outcomes. The FACIT (Folate after Coronary Intervention Trial) trial [9] of folate treatment for those with baseline tHcy concentrations $>13 \mu \mathrm{mol} / \mathrm{L}$ showed a slowing of decline in several cognitive domains and improved performance in episodic memory tests in healthy older people. The VITACOG trial showed that a B vitamin supplement (folic acid, vitamins B6 and B12) in older adults with mild cognitive impairment (MCI) slowed the rate of global [10] and regional brain atrophy [11] and maintained verbal episodic memory, semantic memory, and global cognitive performance (Mini-Mental State Examination, MMSE), but only for those with high baseline tHcy concentrations. After 2 years on B vitamins, more than half of those with elevated tHcy reverted from MCI back to control status, assessed by the Clinical Dementia Rating (CDR) scale [12].

Eating fish rich in long-chain polyunsaturated fatty acids, such as the omega-3 fatty acids eicosapentaenoic acid (EPA) and docosahexaenoic acid (DHA), protects against the onset of dementia [5]. DHA constitutes $30 \%-40 \%$ of long-chain polyunsaturated fatty acids in grey matter cerebral cortex [13]. Intervention trials with DHA and EPA supplements have, like B vitamin trials, given conflicting results on cognitive outcomes $[14,15]$. Some trials suggest that older adults with mild cognitive deficits may benefit more from omega-3 fatty acid supplements than those with Alzheimer's disease [16-19]. Benefits in visuospatial episodic memory were seen after 6 months of supplementation with DHA alone in older adults with mild age-related cognitive decline [18]. Furthermore, in subjects with MCI, EPA plus DHA improved general cognitive performance [17] and letter fluency [20]. A meta-analysis of ten omega-3-fatty acid randomized controlled trials reported a benefit for attention and processing speed in those with cognitive impairment but no dementia [21], while a more recent meta-analysis of studies on the memory domain (episodic, sematic, and working memory) showed that DHA, with or without EPA, contributes to improved memory function in older adults with mild memory complaints [19].

Differences in trial design may account for some of the inconsistency in results [8, 14]. Nutritional interventions to remediate cognition are influenced by factors such as the stage of cognitive decline, the extent of nutritional deficiency of the investigated population, the applied nutrient and its dose, and the duration of the intervention $[22,23]$. Effects will likely be smaller in healthy adults or those with advanced cognitive impairment [24]. It has also been proposed that combinations of different nutrients might be more effective than supplementation with single nutrients $[3,25]$.

In this regard, it is noteworthy that links between omega-3 fatty acids and homocysteine have been suggested [26]. Homocysteine has a role in regulating phospholipid metabolism and omega-3 distribution via the methionine cycle. In turn, B vitamins are important for the synthesis of phospholipids. The question arises: is there a beneficial effect of omega- 3 fatty acids on the disease-modifying actions of B vitamins in MCI? We have already shown that in VITACOG a good omega3 fatty acid status enhances the protective effect of B vitamins on brain atrophy, the primary trail outcome [27]. In this report, we analyze the VITACOG trial data to see if there is an interaction between baseline omega-3 fatty acid status and B vitamin treatment on the secondary cognitive and clinical outcomes of the trial.

\section{MATERIALS AND METHODS}

The recruitment methods and consent process for the VITACOG trial, the inclusion and exclusion criteria, and the neuropsychological tests have been described previously [10, 12]. 266 participants 70 years and over with MCI [28] were randomized either to high dose B vitamins $(n=133)$ or placebo for 2 years. Baseline demographics (age, gender, education) and blood levels of tHcy, vitamin B12, serum folate, vitamin B6, DHA, and EPA were measured, and repeated at the end of the trial period. APOE allele status, depressive symptoms with the geriatric depression scale, and other risk factors for Alzheimer's disease were also assessed [10].

\section{Cognitive testing}

Cognitive tests were administered at baseline and at the 2-year end-point as secondary trial outcomes. 
Some cognitive tests were conducted at more than two time-points during the trial to allow for longitudinal analyses. These included the Hopkins verbal learning test (for verbal episodic memory) with delayed recall (HVLT-DR) using six different versions of the wordlists [29] to minimize a learning effect, and the telephone inventory for cognitive status-modified (TICS-M) [30], which assesses general cognition. The HVLT-DR was conducted face-to-face at baseline and two-year endpoint, but by telephone at 3, 6, 12, and 18 month time points. The TICS-M was assessed at baseline, 15, and 27 months; the latter test was done three months after the treatment ceased. The CDR [31] was administered as a clinical and functional measure to both the participant and informant at baseline and two-year follow-up. See supplement in de Jager et al. [12] for detailed descriptions of the tests and scoring.

\section{Biochemical assays}

Plasma total DHA and EPA were quantified by gas chromatography-mass spectrometry (Focus GC-DSQ II GC-MS system, Thermo Scientific, Hemel Hempstead, UK) as described [27]. The method measures the combined pool of DHA and EPA in plasma, including the free fractions, and those esterified into phospholipids, triglycerides, and cholesteryl esters. In short, $30 \mu \mathrm{L}$ non-fasting plasma was transmethylated using methanolic- $\mathrm{HCl}(3 N)$ at $95^{\circ} \mathrm{C}$ for $2 \mathrm{~h}$. Fatty acid methyl esters were extracted with hexane, and separated on a BPX70 column $(25 \mathrm{~m} \times 0.22 \mathrm{~mm}, 25 \mu \mathrm{m}$ film, SGE, Weiterstadt, Germany). DHA and EPA were quantified by comparison with calibrations curves, corrected for the presence of internal standard, and absolute concentrations were expressed in $\mu \mathrm{mol} / \mathrm{L}$. APOE genotype, plasma tHcy, folate, and vitamin B12 were assayed as previously described [32].

\section{Statistical methods}

The main objective in this study is to show how the baseline concentration of omega-3 fatty acids modifies the effect of B vitamin treatment on cognitive function. The baseline concentrations of combined long-chain omega-3 fatty acids (DHA and EPA), of DHA alone and of EPA alone were divided into tertiles for the placebo group and for the B vitamin group separately. The concentration split-points and numbers in each subgroup are shown in Supplementary Table 4. Interaction terms were tested using the likelihood ratio test and were considered significant if their corresponding $p$ values were $<0.1$. In addition to quantitative interactions, we also statistically investigated for the presence of cross-over interactions. This type of interaction occurs when one treatment is superior for some subgroup(s) of patients and the alternative treatment is superior for another subgroup(s). Please refer to the following papers for examples of cross-over interactions [33, 34].

For each of the cognitive scores, the last follow up measure (24 months or, for TICS-M, 27 months) was statistically modeled as a function of B-vitamin treatment code, baseline omega-3 level (in tertiles) and an interaction term between both. The model adjusted for baseline cognitive score, age, gender, APOE4 status, education, and baseline tHcy. A multiple linear regression model was used for HVLT-DR, TICS-M, and CDR-sum-of-boxes (CDRsob). The CDR overall score was dichotomized into $\mathrm{CDR}=0$ (normal) or $\mathrm{CDR}>0$ (equivalent to MCI and mild Alzheimer's disease) and then used in multiple logistic regression. Longitudinal changes in HVLT-DR, with the six repeated measures (using alternate versions) from baseline through to last follow-up, were analyzed by a linear mixed effect (LME) model. The LME model included main effects of treatment, time, and combined omega-3 levels and two and three-way interactions between these factors. A random intercept and a random slope were included in the LME model. We compared the distribution, across tertiles of omega-3, of demographic and clinical variables at baseline to identify potential confounders that might distort the modifying effect of omega-3 on $B$ vitamin treatment. We dealt with the issue of confounding by using adjusted analysis in the multiple linear regression models [35].

No adjustment procedure for multiplicity was used when testing for multiple hypothesis testing. All analyses were performed using ' $\mathrm{R}$ ' software version 3.0.3.

\section{RESULTS}

\section{Demographics}

The B vitamin treated and placebo groups showed no differences in most demographic variables at baseline, including age, gender ratio, total years of education, APOE4 allele status, smoking, blood pressure, BMI, creatinine, tHcy, folate and vitamin B12 concentrations, DHA, EPA and combined omega3 concentrations, MMSE, HVLT-DR, TICS-M, and CDR scores. The treated group had lower baseline geriatric depression scores than the placebo group (Table 1). Self-reported use of B vitamin supplements and fish oils did not differ between the groups. 
Table 1

Demographic variables at baseline for treatment and placebo groups

\begin{tabular}{|c|c|c|c|}
\hline \multirow[t]{2}{*}{ Variables } & B vitamins $(n=133)$ & Placebo $(n=133)$ & \multirow[t]{2}{*}{$\overline{p \text {-value }}$} \\
\hline & Mean \pm SD & Mean \pm SD & \\
\hline Age & $76.86 \pm 4.89$ & $76.77 \pm 4.90$ & 0.872 \\
\hline School Total & $14.29 \pm 3.35$ & $14.77 \pm 3.38$ & 0.242 \\
\hline \multicolumn{4}{|l|}{ Gender, n (\%) } \\
\hline Male & $86(65)$ & $85(63)$ & \multirow[t]{2}{*}{0.935} \\
\hline Female & $47(35)$ & $49(37)$ & \\
\hline \multicolumn{4}{|l|}{ APOE4+ } \\
\hline Yes & $50(38)$ & $38(28)$ & \multirow[t]{2}{*}{0.140} \\
\hline No & $83(62)$ & $96(72)$ & \\
\hline \multicolumn{4}{|l|}{ Ever Smokers, n (\%) } \\
\hline Yes & $58(44)$ & $68(51)$ & \multirow[t]{2}{*}{0.294} \\
\hline No & $74(56)$ & $65(49)$ & \\
\hline Systolic Blood Pressure & $147.20 \pm 22.41$ & $146.80 \pm 19.82$ & 0.876 \\
\hline Diastolic Blood Pressure & $80.44 \pm 11.19$ & $80.22 \pm 10.87$ & 0.875 \\
\hline Body Mass Index (kg/m²) & $25.76 \pm 3.81$ & $26.27 \pm 4.17$ & 0.302 \\
\hline tHcy & $11.84 \pm 3.40$ & $12.14 \pm 4.03$ & 0.511 \\
\hline Vitamin B12 & $364.86 \pm 166.52$ & $336.73 \pm 105.31$ & 0.101 \\
\hline Serum Folate & $27.37 \pm 17.96$ & $27.23 \pm 18.80$ & 0.951 \\
\hline Creatinine & $95.92 \pm 16.82$ & $97.91 \pm 16.42$ & 0.332 \\
\hline DHA & $309.60 \pm 117.66$ & $319.35 \pm 126.04$ & 0.514 \\
\hline EPA & $206.38 \pm 131.10$ & $218.68 \pm 147.03$ & 0.471 \\
\hline Sum DHA + EPA & $515.98 \pm 235.01$ & $538.02 \pm 363.44$ & 0.471 \\
\hline \multicolumn{4}{|c|}{ Vitamin B supplement use, $\mathrm{n}(\%)$} \\
\hline Yes & $21(16)$ & $25(19)$ & \multirow[t]{2}{*}{0.647} \\
\hline No & $112(84)$ & $109(81)$ & \\
\hline GDS $(0-30)$ & $6.08 \pm 4.43$ & $7.37 \pm 4.94$ & 0.025 \\
\hline TICS-M $(0-39)$ & $24.77 \pm 2.88$ & $24.92 \pm 2.77$ & 0.663 \\
\hline MMSE $(0-30)$ & $28.14 \pm 1.84$ & $28.21 \pm 1.52$ & 0.716 \\
\hline HVLT-DR $(0-12)$ & $7.72 \pm 2.91$ & $7.37 \pm 3.20$ & 0.356 \\
\hline
\end{tabular}

tHcy, total homocysteine, DHA, docosahexaenoic acid; EPA, eicosapentaenoic acid; HVLT-DR, Hopkins Verbal Learning Test delayed recall score; GDS, geriatric depression scale; TICS-M, Telephone Inventory for Cognitive status-modified.

Subjects in the different tertiles of omega-3 showed no differences in most demographic variables at baseline, including age, total years of education, smoking, blood pressure, BMI, and vitamin B12 concentrations (Supplementary Table 1). The highest tertiles of omega-3 were more likely to have a higher proportion of men, lower levels of tHcy, higher levels of folate, and lower levels of creatinine.

\section{Linear regression analyses of final cognitive test performance}

\section{Episodic memory (HVLT-DR) at last follow-up}

The scores for HVLT-DR at the last follow-up (24 months) increased in the $\mathrm{B}$ vitamin treated group across increasing tertiles of combined omega-3 fatty acids, whereas the scores in the placebo group showed negligible change (Fig. 1). The likelihood ratio test showed a significant interaction between

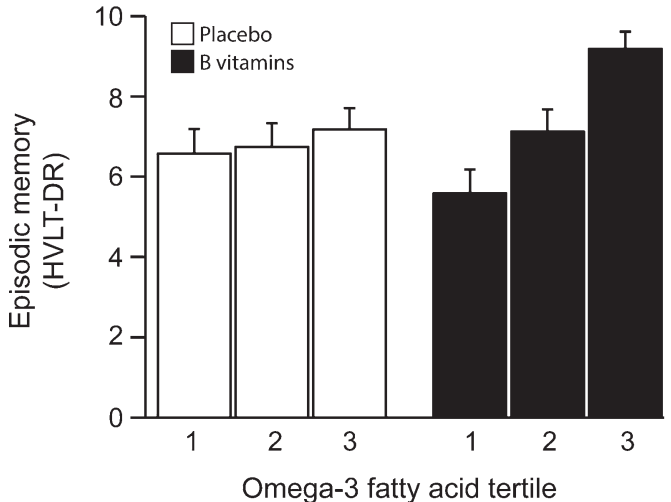

Fig. 1. Episodic memory score after 2 years according to baseline omega- 3 fatty acid concentration. The interaction between omega- 3 tertiles and B vitamin treatment was significant $(p=0.028)$. In the third tertile of the combined omega- 3 fatty acid concentration, the memory score in the B vitamin group was higher than in placebo $(p=0.047)$. In the B vitamin group, memory score in the 3rd tertile of omega- 3 was higher than in the 1 st tertile $(p=0.01)$. See Table 2 . Columns show mean scores and error bars indicate SEM. 
B-vitamin treatment and plasma combined omega-3 fatty acids $\left(p_{\text {interaction }}=0.028\right)$ (Table 2 ). This finding is mainly due to the 2.08 point difference between scores $(p=0.010)$ in the 1 st and 3rd tertile of combined omega- 3 fatty acids. The HVLT-DR score was also significantly higher $(p=0.047)$ in the B vitamin group compared with the placebo group for those with combined omega-3 fatty acids in the 3rd tertile $(>579 \mu \mathrm{mol} / \mathrm{L})$, while at lower omega-3 levels there was no significant difference in performance by treatment code. When HVLT-DR was assessed in relation to tertiles of baseline plasma concentrations of DHA and of EPA separately, similar results were obtained and were particularly significant for DHA (Supplementary Fig. 1 and Supplementary Tables 2 and 3). Thus, for DHA the interaction was significant at $p=0.003$; in the 3rd tertile the average HVLT-DR score was 1.7 points higher in the B vitamin treated group compared with placebo group $(p=0.002)$. A marked concentration-dependent effect of DHA was found in the B vitamin treated group: in the 3rd tertile the average HVLT-DR score was 1.96 points higher than in the 2 nd tertile $(p=0.015)$ and 2.53 points higher than in the 1 st tertile $(p=0.001)$ (Supplementary Table 2).
Global cognition (TICS-M) at last follow-up

The TICS-M scores at the last follow-up (27 months) increased in the B vitamin treated group across increasing tertiles of combined omega-3 fatty acids, whereas the scores in the placebo group were not affected (Fig. 2). The likelihood ratio test showed a significant interaction between $B$ vitamin treatment and combined omega-3 fatty acids ( $p_{\text {interaction }}=0.09$ ). There was also a significant difference in the treatment effects between the 3 rd and the 1st tertile of combined omega-3 fatty acids (difference $=2.85$ points, $p=0.035$ ) with higher scores for those in the 3rd tertile (Fig. 2 and Supplementary Table 1).

When TICS-M was assessed in relation to tertiles of baseline plasma concentrations of DHA and EPA separately, only higher concentrations of DHA significantly enhanced the cognitive effects of B vitamins, $p_{\text {interaction }}=0.098$ (Supplementary Fig. 2 and Supplementary Tables 2 and 3). In the 3rd DHA tertile, there was a significant effect of $B$ vitamin treatment on TICS-M $(p=0.041)$ and in the B vitamin treatment group there was a concentration-dependent effect of DHA with a 2.78 point higher TICS-M score in the 3rd tertile compared with the 1 st tertile $(p=0.039)$ (Supplementary Table 2).

Table 2

Results of the fit of the linear regression model for cognitive and clinical outcomes and concentrations of combined omega- 3 fatty acids $(\mathrm{DHA}+\mathrm{EPA})$

\begin{tabular}{|c|c|c|c|c|c|c|c|}
\hline & \multicolumn{3}{|c|}{ Treatment Effect $^{1}$} & \multirow{2}{*}{$\begin{array}{c}\text { Overall interaction }^{3} \\
p \text { value }^{4}\end{array}$} & \multicolumn{3}{|c|}{ Tertiles pairwise comparisons } \\
\hline & Crude & Adjusted & $p$ value $^{2}$ & & ${ }^{5} p_{1 \text { st vs } 2 \text { nd }}$ & $p_{1 \text { st vs } 3 \text { rd }}$ & $p_{2 \text { nd vs } 3 \text { rd }}$ \\
\hline HVLT-DR & & & & 0.028 & & & \\
\hline Tertile 1 & -0.7 & -0.94 & 0.097 & & $\operatorname{diff}=1.36$ & $\operatorname{diff}=2.08$ & $\operatorname{diff}=0.72$ \\
\hline Tertile 2 & 0.4 & 0.42 & 0.44 & & $p=0.087$ & $p=0.010$ & $p=0.37$ \\
\hline Tertile 3 & 2 & 1.14 & 0.047 & & & & \\
\hline TICS-M & & & & 0.09 & & & \\
\hline Tertile 1 & -1.6 & -1.07 & 0.25 & & $\operatorname{diff}=1.62$ & $\operatorname{diff}=2.85$ & $\operatorname{diff}=1.23$ \\
\hline Tertile 2 & 0.4 & 0.55 & 0.56 & & $p=0.22$ & $p=0.035$ & $p=0.36$ \\
\hline Tertile 3 & 2.8 & 1.78 & 0.062 & & & & \\
\hline CDR (OR \& 95\% CI) & & & & 0.13 & & & \\
\hline Tertile & $1.99(0.64,6.44)$ & $1.50(0.48,4.79)$ & 0.48 & & diff in & diff in $\log$ & diff in $\log$ \\
\hline Tertile 2 & $0.41(0.12,1.31)$ & $0.43(0.13,1.36)$ & 0.15 & & $\log \mathrm{OR}=-1.25$ & $\mathrm{OR}=-1.57$ & $\mathrm{OR}=-0.32$ \\
\hline Tertile 3 & $0.35(0.11,1.08)$ & $0.31(0.10,0.95)$ & 0.043 & & $p=0.13$ & $p=0.053$ & $p=0.69$ \\
\hline CDRsob & & & & 0.35 & & & \\
\hline Tertile 1 & 0.26 & -0.03 & 0.92 & & $\operatorname{diff}=-0.36$ & $\operatorname{diff}=-0.50$ & $\operatorname{diff}=-0.14$ \\
\hline Tertile 2 & -0.34 & -0.38 & 0.16 & & $p=0.34$ & $p=0.17$ & $p=0.69$ \\
\hline Tertile 3 & -0.66 & -0.53 & 0.040 & & & & \\
\hline
\end{tabular}

${ }^{1}$ Defined as the average score in treated minus the average score in placebo for HVLT-DR, TICS-M and CDRsob. For CDR it is the OR ratio for a worse outcome comparing treated to placebo. The crude estimate uses the raw data without any statistical modeling. The adjusted treatment effect was obtained by using statistical modeling and adjusting for baseline cognitive score, age, gender, APOE4 status, education, and baseline tHcy. ${ }^{2}$ This is the $p$-value for testing the null hypothesis of no treatment effect within a fixed tertile. This applies to adjusted analysis only. ${ }^{3}$ Overall interaction tests the null hypothesis that treatment effects in $1 \mathrm{st}, 2 \mathrm{nd}$ and $3 \mathrm{rd}$ tertiles are all the same. ${ }^{4}$ This is the $p$-value for testing the null hypothesis of no overall interaction. ${ }^{5} \mathrm{p}_{1 \mathrm{st} \text { vs } 2 \mathrm{nd}}$ is the $p$-value for testing the null hypothesis that treatment effects in $1 \mathrm{st}$ and $2 \mathrm{rd}$ tertiles are the same. The same applies for $\mathrm{p}_{1 \mathrm{st} \text { vs } 3 \mathrm{rd}}$ and $\mathrm{p}_{2 \mathrm{nd} \mathrm{vs} 3 \mathrm{rd}}$. 


\section{Clinical dementia rating: CDR (binary variable) at last follow-up}

The proportion of participants who had a CDR overall score of $>0$ (worse outcome) at the 24 month follow-up is shown in Fig. 3A. In the 3rd tertile of baseline combined omega-3 fatty acids only $33 \%$ of those on B vitamin treatment had CDR scores $>0$ compared with $59 \%$ in the placebo group. Also, in the $3 \mathrm{rd}$ tertile, B vitamins markedly reduced the risk of having CDR scores $>0$ (adjusted OR 0.31, 95\% CI 0.10, $0.95 ; p=0.043$ ) (Table 2). There was a trend for a

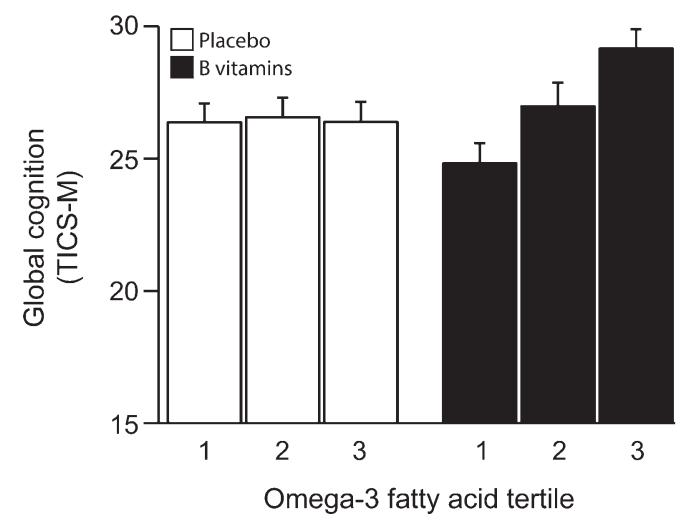

Fig. 2. Global cognition after 2 years according to baseline omega-3 fatty acid concentration. The interaction between omega- 3 tertiles and $\mathrm{B}$ vitamin treatment was significant $(p=0.09)$. In the $\mathrm{B}$ vitamin group, global cognition score in the 3 rd tertile of omega- 3 was higher than in 1 st tertile $(p=0.035)$. See Table 2. Columns show mean scores and error bars indicate SEM.

(A)

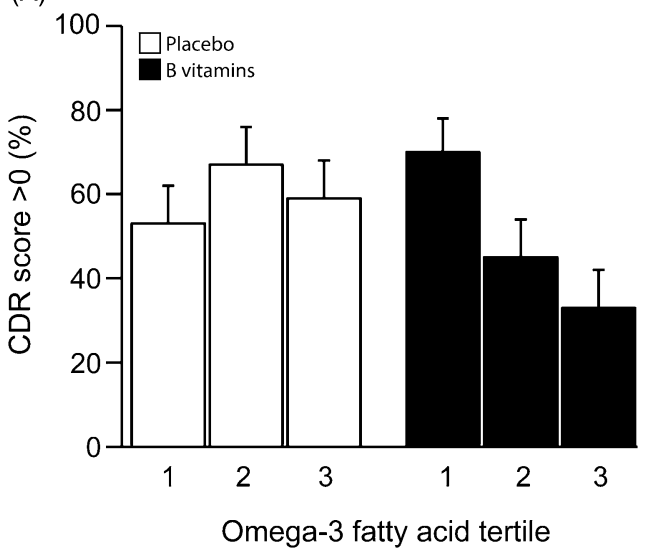

concentration-dependent effect of combined omega-3 fatty acids: the proportion scoring $>0$ in the B vitamin group declined from $70 \%$ to $33 \%$ from the 1 st tertile to the 3rd tertile $(p=0.053)$.

Significant interaction effects were also found for DHA ( pinteraction $=0.097$ ), with an adjusted odds ratio of $0.26(95 \%$ CI $0.08,0.81)$ for a worse CDR, comparing $\mathrm{B}$ vitamin treated versus placebo in the $3 \mathrm{rd}$ tertile of DHA $(p=0.022)$ (Supplementary Table 2 and Supplementary Fig. 3A). There was a concentrationdependent effect of DHA in the B vitamin group with a greater improvement in the 3rd than in the 1st tertile ( $p=0.034)$ (Supplementary Table 2). The effects of EPA on the CDR showed similar patterns but did not reach significance (Supplementary Table 3 and Supplementary Fig. 3B).

\section{Clinical dementia rating: CDR-sum-of-boxes at last follow-up}

The average scores for CDRsob are shown in Fig. 3B. The likelihood ratio test failed to show a significant overall interaction at $\alpha$ level $10 \%$ between B-vitamin treatment and plasma combined omega-3 fatty acids on CDRsob ( $\left.p_{\text {interaction }}=0.35\right)$. However, there was a significant difference in the average CDRsob score in the 3rd omega-3 tertile between $B$ vitamin and placebo groups: average difference $=-$ $0.53, p=0.040$ (Table 2). For DHA alone there was a significant effect of $\mathrm{B}$ vitamin treatment on CDRsob in the third tertile of DHA $(p=0.03)$. For EPA alone,

(B)

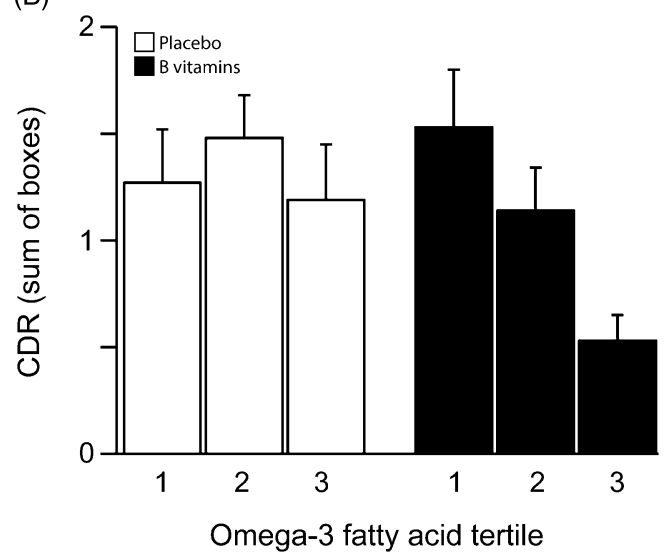

Fig. 3. (A) Clinical Dementia Rating score after 2 years according to baseline omega-3 fatty acid concentration. The interaction between omega-3 tertiles and B vitamin treatment did not reach significance $(p=0.13)$. In the 3rd tertile of combined omega-3 fatty acids, the percent of subjects with CDR $>0$ was lower in the B vitamin group than in the placebo group $(p=0.043)$. See Table 2 . Columns show mean scores and error bars indicate SEM. (B) Clinical Dementia Rating sum of boxes score after 2 years according to baseline omega-3 fatty acid concentration. The interaction between omega-3 tertiles and B vitamin treatment was not significant $(p=0.35)$. In the 3 rd tertile of combined omega-3 fatty acids, the CDRsob score was lower in the B vitamin group than in the placebo group $(p=0.040)$. See Table 2. Columns show mean scores and error bars indicate SEM. 

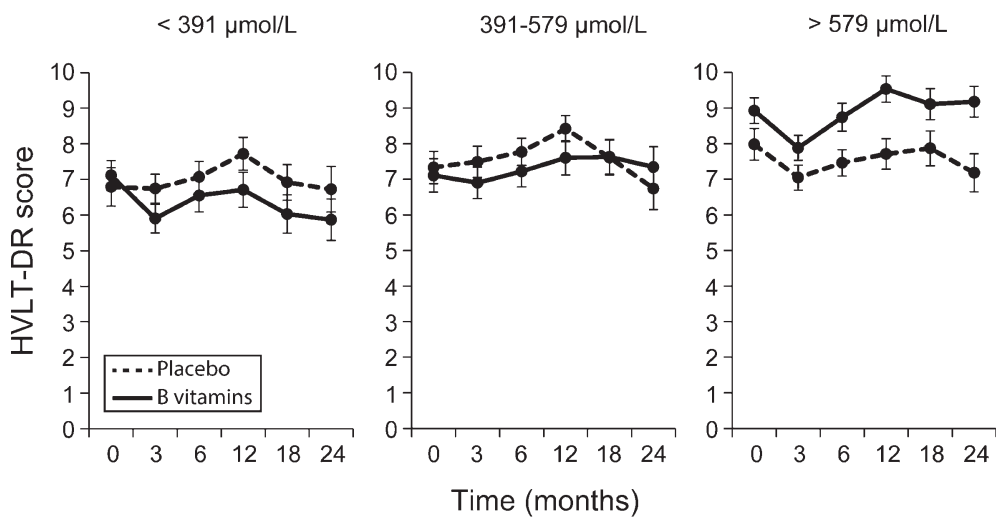

Fig. 4. Longitudinal scores of episodic memory (HVLT-DR) across tertiles of combined omega-3 fatty acids. Solid line: B vitamin group; dashed line: placebo group. Ranges of the tertiles are given in Supplementary Table 4. The likelihood ratio test for interaction between B vitamin treatment and combined omega-3 tertiles was significant at an alpha level 10\% ( $p=0.086$, Supplementary Table 6). In the 3rd tertile of combined omega-3, the average HVLT-DR significantly increased in the B vitamin group by 0.46 points per year of follow-up ( $p=0.013)$ compared to no significant change in the placebo group (Supplementary Table 6). Error bars represent SEM.

the pattern was similar but did not reach significance (Supplementary Tables 2 and 3; Supplementary Fig. 4A, B).

\section{Longitudinal analysis of episodic memory (HVLT-DR)}

The results of fitting the linear mixed effects model on the repeated measures of HVLT-DR showed that the effect of B-vitamin treatment depended on the baseline plasma concentration of combined omega-3 fatty acids and DHA alone (Fig. 4, Supplementary Fig. 6A, Supplementary Table 6). The likelihood ratio test for interaction between B vitamin treatment and combined omega-3 was significant at an alpha level $10 \%$ $(p=0.086)$ and also for interaction between B vitamin treatment and DHA $(p=0.025)$, but did not reach significance for EPA (Supplementary Table 6). The modifying effect of omega-3 was mainly characterized by different slopes in placebo and B vitamin group in the 3 rd tertile compared to the 1 st tertile. For example, in the 3rd tertile of combined omega-3, the average HVLT-DR significantly increased in the B vitamin group by 0.46 points per year of follow-up ( $p=0.013$ ) compared to no significant change in the placebo group $(p=0.91)$. However, in the 1 st tertile, there were no significant changes in HVLT-DR in either placebo $(p=0.36)$ or B-vitamin groups $(p=0.44)$. The same pattern occurred for the third tertile of DHA (Supplementary Fig. 5A) with an increase in HVLT-DR score of 0.46 points per year in the B vitamin group $(p=0.009)$, but no significant change in the placebo group (Supplementary Table 6). The pattern for EPA was similar to that for DHA (Supplementary Fig. 5B) but did not reach significance (Supplementary Table 6).

\section{Testing for qualitative or cross-over interactions}

We observed a consistent trend for subjects in the lowest tertile of omega-3: B-vitamin treatment, compared with placebo, appeared to be associated with lower cognitive scores. However, there is no evidence for any cross-over interactions as shown in Supplementary Table 5. This means that treatment effects are not in opposite directions across tertiles of omega-3. Although this observation was not significant on analysis of cross-over interactions, it will be important to examine this possibility in other observational studies and clinical trials. Residual confounding cannot be excluded.

\section{DISCUSSION}

This study revealed significant beneficial effects of higher baseline omega-3 fatty acid concentrations on certain cognitive and clinical outcomes of B vitamin treatment in older persons with MCI, both crosssectionally at the study end, and longitudinally for episodic memory, over the 2-year intervention period. The cross-sectional regression analyses for all cognitive and clinical outcomes tested showed similar patterns. For participants with high baseline levels of omega-3 fatty acids, the B vitamin treated group performed better than placebo, while those with low baseline omega-3 fatty acids did not benefit from B vitamin supplementation. In general, the effects were 
more significant for DHA alone than for EPA or combined omega-3 fatty acids, especially for the HVLT-DR and TICS-M. However, due to the small number of participants and a considerable co-variation between DHA and EPA, the present data set is not ideal for separating effects between the two fatty acids. Overall, DHA and EPA showed similar patterns on all cognitive outcomes (see Supplementary Figures). These results should be used for hypothesis generation and optimization of future trials.

As mentioned in the Introduction, trials of both omega-3 fatty acids and, separately, of B vitamins have given conflicting results regarding cognitive outcomes. Nevertheless, some previous studies have shown benefits of omega- 3 fatty acid treatment alone on various types of memory [14, 19], attention and processing speed [21], as well as global cognition [17], while trials with B vitamins have shown benefits on episodic memory, verbal fluency, and global cognition in people with high baseline tHcy [9, 12]. We find here that this beneficial effect of B vitamin treatment on cognition only clearly occurs in those with a good omega-3 fatty acid status. A similar interaction between omega-3 fatty acid status and B vitamin treatment was found in VITACOG for the slowing of brain atrophy rate [27]. Our previous findings revealed that cognitive scores including the HVLT-DR and TICS-M decline more rapidly in those with the most brain atrophy [36]. We discuss below the likely causal links between cognitive scores and regional brain atrophy.

On the whole, trials on the efficacy of B vitamin supplementation for cognitive impairment have produced variable and sometimes negative results (see Introduction). Our results lead us to suggest that the variable outcomes might in part be related to different omega-3 fatty acid status in the trial participants, either due to diet or supplement intake. If intake is not monitored at baseline and controlled for in the randomization of participants to treatment, omega-3 status may confound the results. Future trials of B vitamin treatment should accordingly control for omega- 3 fatty acid status. The different cognitive outcomes used may also account for variable outcomes in clinical trials. Manders et al. [24] found that general cognitive tests such as the MMSE often did not show effects for nutrients. But positive results were obtained when domain-specific tests were used, especially for fluid rather than crystallized ability, such as information processing. We found significant results for the episodic memory domain (HVLT-DR) and for general cognition with the TICS-M. However, the TICS-M contains more memory items than the
MMSE and is a more sensitive test for cognitive decline [30].

There has been one randomized trial with a combination of $\mathrm{B}$ vitamins and omega- 3 fatty acids in which cognition was assessed [37]. This was a secondary prevention trial in patients with cardiovascular disease, but only one cognitive assessment was done, at the end of the trial, and so it was not possible to study the effect of the treatment upon cognitive decline. Overall, no significant treatment effects were found on the final cognitive test scores, apart from a higher score in temporal orientation in those with a history of ischemic stroke and a lower score in semantic memory in those with a history of heart disease. This trial was not designed to study cognitive decline, and used doses of nutrients at dietary levels rather than pharmacological levels; it is thus not suitable for answering the question of whether omega-3 fatty acids enhance the slowing effect of $B$ vitamins on cognitive decline. A randomized trial should now be performed with high dose combinations of these supplements in people who will experience cognitive decline during the trial period, such as those with MCI.

The findings from VITACOG, here on cognition and earlier on brain atrophy [27], are evidence of an interaction between two different classes of nutrients, B vitamins and omega-3 fatty acids, in people with MCI. Possible mechanisms for this interaction have been discussed in our earlier report [27] and include the hypothesis that B vitamins facilitate the formation of phosphatidylcholine (PC) enriched in omega-3 fatty acids from phosphatidylethanolamine (Fig. 5) and hence the transport of omega- 3 fatty acids into the brain $[26,38]$. PC synthesized by this B vitamin-

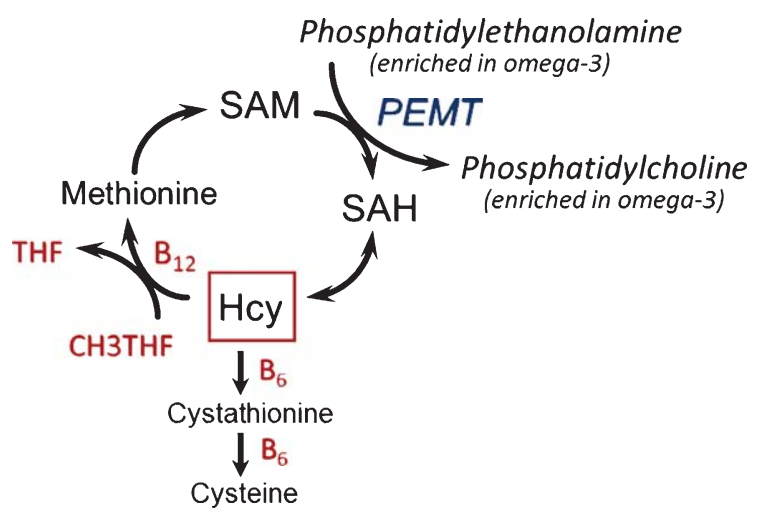

Fig. 5. Metabolic interactions in the homocysteine methylation cycle with omega-3 fatty acids. Hcy, homocysteine; $P E M T$, phosphatidylethanolamine N-methyl transferase; SAH, Sadenosylhomocysteine; SAM, S-adenosylmethionine; THF, tetrahydrofolate. 
dependent pathway has been shown to contain higher levels of polyunsaturated fatty acids, including omega3 , compared with the alternative CDP-choline pathway $[39,40]$. Selley [26] found that the raised plasma Sadenosylhomocysteine in patients with Alzheimer's disease was associated with decreased erythrocyte concentrations of PC and with increased concentrations of phosphatidylethanolamine; he therefore suggested that raised homocysteine inhibits phosphatidylethanolamine methyltransferase. Selley [26] also found that the PC in Alzheimer's disease erythrocytes was relatively depleted in DHA. It is noteworthy that decreased levels of polyunsaturated fatty acids in PC occur in the brain in Alzheimer's disease [38]. Also, low levels of omega-3 fatty acids (DHA and EPA) in plasma PC are a risk factor for dementia [41] and have been found in plasma PC in patients with Alzheimer's disease [42]. Animal experiments are consistent with the above hypothesis: rats made deficient in folate had impaired spatial memory performance and showed a halving in brain PC concentration and an increase in brain phosphatidylethanolamine [43]. Notably, the memory deficit and the decline in brain PC were both reversed by treatment of the rats with methionine. It remains to be shown whether the depletion of brain PC in folate deficiency is associated with a loss of polyunsaturated fatty acids.

The striking agreement between the results on the rate of brain atrophy and on cognitive decline in VITACOG raises the question of whether the two outcomes are causally linked. We have previously addressed this question by mediation analysis [11]. We found associations between cognitive decline and loss of gray matter in specific brain regions. Worsening of CDRsob and MMSE scores was associated with gray matter loss, most pronounced bilaterally in the amygdalo-hippocampal complex and entorhinal cortex. Decreases in HVLT-delayed recall and category fluency scores were associated with increased gray matter loss in the left hippocampus and entorhinal cortex. These gray matter regions involved in cognitive decline also showed a marked reduction of atrophy with B-vitamin treatment in subjects with high tHcy levels. We found that the optimal Bayesian network model explaining our data indicated the following causal chain of events: B vitamin treatment (mainly vitamin B12) caused a fall in tHcy levels, which reduced the rate of gray matter atrophy, which, in turn, delayed cognitive decline (HVLT-DR, category fluency, CDRsob) [11]. It would be of interest to see what the effect would be of the inclusion of omega-3 fatty acid status in these analyses.
A limitation of our study is that omega- 3 fatty acids were not supplemented during the trial, so the findings only relate to baseline levels. Another limitation, which may account for the variability between subgroups, was the small number of participants in some of the subgroups. The small numbers also prevented us from looking for modifying factors, such as the possible interaction between the effects of omega- 3 fatty acids and APOE4 status [44].

In conclusion, when plasma omega- 3 fatty acid concentrations are low, B vitamin treatment does not slow cognitive decline in people with MCI. In contrast, when omega-3 fatty acid levels are in the upper range of normal, the slowing effects of B vitamins on both brain atrophy [27] and cognitive decline are enhanced. We suggest that the effects of this interaction between the two nutrients on brain atrophy and cognition is consistent with the view that they slow down the disease process in MCI. These results may have public health implications. It will be important to test in a clinical trial whether the administration of these two nutrients will delay the conversion from MCI to dementia.

\section{ACKNOWLEDGMENTS}

We are grateful for contributions from the OPTIMA team, in particular the nurses, and to a principal investigator for the study, Professor Robin Jacoby, and to all the volunteers who took part in the trial. We thank Carole Johnston, Cynthia Prendergast and Cheryl Turner for laboratory analyses. This study was supported by grants from UK MRC, Charles Wolfson Charitable Trust, Norman Collisson Foundation and Norwegian Research Council.

Authors' disclosures available online (http://j-alz. com/manuscript-disclosures/15-0777r1).

\section{SUPPLEMENTARY MATERIAL}

The supplementary material is available in the electronic version of this article: http://dx.doi.org/ 10.3233/JAD-150777.

\section{REFERENCES}

[1] Gómez-Pinilla F (2008) Brain foods: The effects of nutrients on brain function. Nat Rev Neurosci 9, 568-578.

[2] Gillette-Guyonnet S, Secher M, Vellas B (2013) Nutrition and neurodegeneration: Epidemiological evidence and challenges for future research. Br J Clin Pharmacol 75, 738-755.

[3] Barberger-Gateau P (2014) Nutrition and brain aging: How can we move ahead? Eur J Clin Nutr 68, 1245-1249. 
[4] Hooshmand B, Polvikoski T, Kivipelto M, Tanskanen M, Myllykangas L, Erkinjuntti T, Makela M, Oinas M, Paetau A, Scheltens P, van Straaten EC, Sulkava R, Solomon A (2013) Plasma homocysteine, Alzheimer and cerebrovascular pathology: A population-based autopsy study. Brain 136, 2707-2716.

[5] Beydoun MA, Beydoun HA, Gamaldo AA, Teel A, Zonderman AB, Wang Y (2014) Epidemiologic studies of modifiable factors associated with cognition and dementia: Systematic review and meta-analysis. BMC Public Health 14, 643.

[6] Ford AH, Almeida OP (2012) Effect of homocysteine lowering treatment on cognitive function: A systematic review and meta-analysis of randomized controlled trials. J Alzheimers Dis 29, 133-149.

[7] Clarke R, Bennett D, Parish S, Lewington S, Skeaff M, Eussen SJ, Lewerin C, Stott DJ, Armitage J, Hankey GJ, Lonn E, Spence JD, Galan P, de Groot LC, Halsey J, Dangour AD, Collins R, Grodstein F, on behalf of the BVTTC (2014) Effects of homocysteine lowering with B vitamins on cognitive aging: Meta-analysis of 11 trials with cognitive data on 22,000 individuals. Am J Clin Nutr 100, 657-666.

[8] McCaddon A, Miller JW (2015) Assessing the association between homocysteine and cognition: Reflections on Bradford Hill, meta-analyses and causality. Nutr Rev 73, 723-735.

[9] Durga J, van Boxtel MP, Schouten EG, Kok FJ, Jolles J, Katan MB, Verhoef P (2007) Effect of 3-year folic acid supplementation on cognitive function in older adults in the FACIT trial: A randomised, double blind, controlled trial. Lancet 369, 208-216.

[10] Smith AD, Smith SM, de Jager CA, Whitbread P, Johnston C, Agacinski G, Oulhaj A, Jacoby R, Refsum H (2010) Homocysteine-lowering by $\mathrm{B}$ vitamins slows the rate of accelerated brain atrophy in mild cognitive impairment. A randomized controlled trial. PLoS One 5, e12244.

[11] Douaud G, Refsum H, de Jager CA, Jacoby R, Nichols TE, Smith SM, Smith AD (2013) Preventing Alzheimer's diseaserelated gray matter atrophy by B-vitamin treatment. Proc Natl Acad Sci U S A 110, 9523-9528.

[12] de Jager CA, Oulhaj A, Jacoby R, Refsum H, Smith AD (2012) Cognitive and clinical outcomes of homocysteinelowering B-vitamin treatment in mild cognitive impairment: A randomized controlled trial. Int J Geriatr Psychiatry 27, 592-600.

[13] Lauritzen L, Hansen HS, Jorgensen MH, Michaelsen KF (2001) The essentiality of long chain n-3 fatty acids in relation to development and function of the brain and retina. Prog Lipid Res 40, 1-94.

[14] Dacks PA, Shineman DW, Fillit HM (2013) Current evidence for the clinical use of long-chain polyunsaturated n-3 fatty acids to prevent age-related cognitive decline and Alzheimer's disease. J Nutr Health Aging 17, 240-251.

[15] Chew EY, Clemons TE, Agron E, Launer LJ, Grodstein F, Bernstein PS, Age-Related Eye Disease Study 2 Research G (2015) Effect of omega-3 fatty acids, Lutein/Zeaxanthin, or other nutrient supplementation on cognitive function: The AREDS2 randomized clinical trial. JAMA 314, 791-801.

[16] Freund-Levi Y, Eriksdotter-Jonhagen M, Cederholm T, Basun H, Faxen-Irving G, Garlind A, Vedin I, Vessby B, Wahlund LO, Palmblad J (2006) Omega-3 fatty acid treatment in 174 patients with mild to moderate Alzheimer disease: OmegAD study: A randomized double-blind trial. Arch Neurol 63, 14021408.

[17] Chiu CC, Su KP, Cheng TC, Liu HC, Chang CJ, Dewey ME, Stewart R, Huang SY (2008) The effects of omega-3 fatty acids monotherapy in Alzheimer's disease and mild cog- nitive impairment: A preliminary randomized double-blind placebo-controlled study. Prog Neuropsychopharmacol Biol Psychiatry 32, 1538-1544.

[18] Yurko-Mauro K, McCarthy D, Rom D, Nelson EB, Ryan AS, Blackwell A, Salem N Jr, Stedman M (2010) Beneficial effects of docosahexaenoic acid on cognition in age-related cognitive decline. Alzheimers Dement 6, 456-464.

[19] Yurko-Mauro K, Alexander DD, Van Elswyk ME (2015) Docosahexaenoic acid and adult memory: A systematic review and meta-analysis. PLoS One 10, e0120391.

[20] Sinn N, Milte CM, Street SJ, Buckley JD, Coates AM, Petkov J, Howe PR (2012) Effects of n-3 fatty acids, EPA v. DHA, on depressive symptoms, quality of life, memory and executive function in older adults with mild cognitive impairment: A 6-month randomised controlled trial. Br J Nutr 107, 1682-1693.

[21] Mazereeuw G, Lanctot KL, Chau SA, Swardfager W, Herrmann N (2012) Effects of omega-3 fatty acids on cognitive performance: A meta-analysis. Neurobiol Aging 33, 1482 e1417-1429.

[22] Morris MC, Tangney CC (2011) A potential design flaw of randomized trials of vitamin supplements. JAMA 305, 13481349.

[23] de Jager CA, Dye L, de Bruin EA, Butler L, Fletcher J, Lamport DJ, Latulippe ME, Spencer JP, Wesnes K (2014) Criteria for validation and selection of cognitive tests for investigating the effects of foods and nutrients. Nutr Rev 72, 162-179.

[24] Manders M, de Groot LC, van Staveren WA, WoutersWesseling W, Mulders AJ, Schols JM, Hoefnagels WH (2004) Effectiveness of nutritional supplements on cognitive functioning in elderly persons: A systematic review. J Gerontol A Biol Sci Med Sci 59, 1041-1049.

[25] Kamphuis PJ, Scheltens P (2010) Can nutrients prevent or delay onset of Alzheimer's disease? J Alzheimers Dis 20, 765775.

[26] Selley ML (2007) A metabolic link between S-adenosylhomocysteine and polyunsaturated fatty acid metabolism in Alzheimer's disease. Neurobiol Aging 28, 1834-1839.

[27] Jernerén F, Elshorbagy AK, Oulhaj A, Smith SM, Refsum H, Smith AD (2015) Brain atrophy in cognitively impaired elderly: The importance of long-chain omega-3 fatty acids and $\mathrm{B}$ vitamin status in a randomized controlled trial. Am J Clin Nutr 102, 215-221.

[28] Petersen RC (2004) Mild cognitive impairment as a diagnostic entity. J Intern Med 256, 183-194.

[29] Brandt J (1991) The Hopkins Verbal Learning Test: Development of a new memory test with six equivalent forms. Clin Neuropsychol 5, 125-142.

[30] de Jager CA, Budge MM, Clarke R (2003) Utility of TICS-m for the assessment of cognitive function in older adults. Int $J$ Geriatr Psychiatry 18, 318-324.

[31] Morris JC (1993) The Clinical Dementia Rating (CDR): Current version and scoring rules. Neurology 43, 2412-2414.

[32] Vogiatzoglou A, Refsum H, Johnston C, Smith SM, Bradley KM, de Jager C, Budge MM, Smith AD (2008) Vitamin B12 status and rate of brain volume loss in community-dwelling elderly. Neurology 71, 826-832.

[33] Gail M, Simon R (1985) Testing for qualitative interactions between treatment effects and patient subsets. Biometrics $\mathbf{4 1}$ 361-372.

[34] Pan G, Wolfe DA (1997) Test for qualitative interaction of clinical significance. Stat Med 16, 1645-1652.

[35] Maldonado G, Greenland S (1993) Simulation study of confounder-selection strategies. Am J Epidemiol 138, 923936. 
[36] de Jager CA (2014) Critical levels of brain atrophy associated with homocysteine and cognitive decline. Neurobiol Aging 35(Suppl 2), S35-S39.

[37] Andreeva VA, Kesse-Guyot E, Barberger-Gateau P, Fezeu L, Hercberg S, Galan P (2011) Cognitive function after supplementation with $\mathrm{B}$ vitamins and long-chain omega-3 fatty acids: Ancillary findings from the SU.FOL.OM3 randomized trial. Am J Clin Nutr 94, 278-286.

[38] Liu JJ, Green P, John Mann J, Rapoport SI, Sublette ME (2015) Pathways of polyunsaturated fatty acid utilization: Implications for brain function in neuropsychiatric health and disease. Brain Res 1597, 220-246.

[39] DeLong CJ, Shen YJ, Thomas MJ, Cui Z (1999) Molecular distinction of phosphatidylcholine synthesis between the CDP-choline pathway and phosphatidylethanolamine methylation pathway. J Biol Chem 274, 29683-29688.

[40] Pynn CJ, Henderson NG, Clark H, Koster G, Bernhard W, Postle AD (2011) Specificity and rate of human and mouse liver and plasma phosphatidylcholine synthesis analyzed in vivo. J Lipid Res 52, 399-407.

[41] Schaefer EJ, Bongard V, Beiser AS, Lamon-Fava S, Robins SJ, Au R, Tucker KL, Kyle DJ, Wilson PW, Wolf PA (2006)
Plasma phosphatidylcholine docosahexaenoic acid content and risk of dementia and Alzheimer disease: The Framingham Heart Study. Arch Neurol 63, 1545-1550.

[42] Whiley L, Sen A, Heaton J, Proitsi P, Garcia-Gomez D, Leung R, Smith N, Thambisetty M, Kloszewska I, Mecocci P, Soininen H, Tsolaki M, Vellas B, Lovestone S, Legido-Quigley C, AddNeuroMed C (2014) Evidence of altered phosphatidylcholine metabolism in Alzheimer's disease. Neurobiol Aging 35, 271-278.

[43] Troen AM, Chao WH, Crivello NA, D'Anci KE, ShukittHale B, Smith DE, Selhub J, Rosenberg IH (2008) Cognitive impairment in folate-deficient rats corresponds to depleted brain phosphatidylcholine and is prevented by dietary methionine without lowering plasma homocysteine. J Nutr 138, 2502-2509.

[44] Barberger-Gateau P, Samieri C, Feart C, Plourde M (2011) Dietary omega 3 polyunsaturated fatty acids and Alzheimer's Disease: Interaction with apolipoprotein E genotype. Curr Alzheimer Res 8, 479-491. 